\title{
On tangle decompositions of twisted torus knots
}

\author{
by
}

\section{Kanji Morimoto}

\author{
Department of IS and Mathematics, Konan University \\ Okamoto 8-9-1, Higashi-Nada, Kobe 658-8501, Japan \\ e-mail : morimoto@konan-u.ac.jp
}

\begin{abstract}
In the present paper, we will show that for any integer $n>0$ there are infinitely many twisted torus knots with $n$-string essential tangle decompositions.
\end{abstract}

Keywords and phrases : twisted torus knots, tangle decompositions

2010 Mathematics Subject Classification : 57M25, 57M27

\section{Introduction}

Let $p, q, r, s$ be integers with $p>r>1, q>0, \operatorname{gcd}(p, q)=1$, and let $T(p, q)$ be the torus knot of type $(p, q)$ in $S^{3}$. For the definition of torus knots $T(p, q)$ we refer to [9]. Add $s$ times full twists on mutually parallel $r$-strands in $T(p, q)$. Then according as [2], we call the knot obtained by this operation a twisted torus knot of type $(p, q ; r, s)$ and denote it by $T(p, q ; r, s)$ as illustrated in Figure 1 .

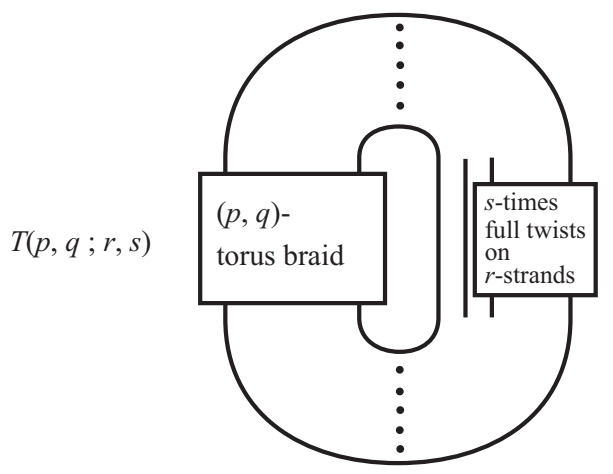

Figure 1

Recently, several interesting results on twisted torus knots have been gotten ([3], $[4],[5],[6],[7],[8])$. In particular, we have shown in [6] that there are infinitely many composite twisted torus knots. In the present paper, as an extension of the result in [6], we will construct infinitely many twisted torus knots with $n$-string essential tangle decompositions for any integer $n>0$. In fact we will show : 
Theorem 1.1. Let $e>0, k_{1}>1, k_{2}>1, x_{1}>0, x_{2}>0$ be integers with $\operatorname{gcd}\left(x_{1}, x_{2}\right)=1$. Put

$$
\begin{aligned}
& p=\left((e+1)\left(k_{1}+k_{2}-1\right)+1\right) x_{1}+(e+1) x_{2}, \\
& q=\left(e\left(k_{1}+k_{2}-1\right)+1\right) x_{1}+e x_{2}, \\
& r=\left((e+1)\left(k_{1}+k_{2}-1\right)-k_{1}+2\right) x_{1}+e x_{2} \text { and } \\
& s=-1 .
\end{aligned}
$$

Then we have :

(1) $T(p, q ; r, s)$ has an $x_{1}$-string essential tangle decomposition.

(2) The decomposition is obtained by the $x_{1}$-string fusion of the torus knot $T\left(\left(k_{1}-\right.\right.$ 1) $\left.x_{1}+x_{2}, e\left(\left(k_{1}-1\right) x_{1}+x_{2}\right)+x_{1}\right)$ and the torus link $T\left(k_{2} x_{1},-\left((e+1) k_{2}+1\right) x_{1}\right)$.

(3) $T(p, q ; r, s)$ has an essential torus in the exterior whose companion is the torus knot $T\left(k_{2},-(e+1) k_{2}-1\right)$.

Therefore, for any integer $n>0$, by putting $x_{1}=n$ we get infinitely many twisted torus knots with n-string essential tangle decompositions.

Example 1.2. Put $e=1, k_{1}=k_{2}=2, x_{1}=2, x_{2}=3$. Then by Theorem 1.1, we see that $T(20,11 ; 15,-1)$ has a 2 -string essential tangle decomposition which is obtained by the 2 -string fusion of $T(5,7)$ and $T(4,-10)$ as in Figure 2 (c.f. Example 3.3). By tubing the decomposing 2 -sphere along the torus link $T(4,-10)$, we have an essential torus whose companion is the torus knot $T(2,-5)$.

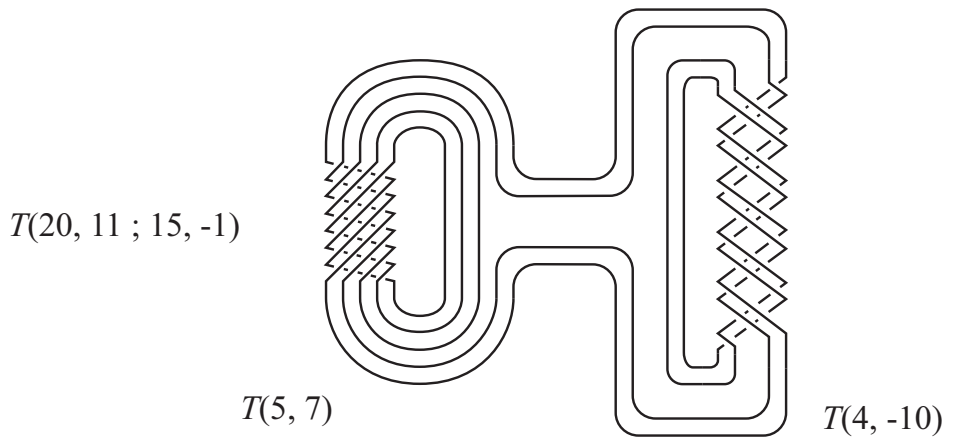

Figure 2

Remark 1.3. Suppose $x_{1}=1$ in Theorem 1.1. Then by putting $k_{1}^{\prime}=k_{1}+x_{2}-1$ and $k_{2}^{\prime}=k_{2}$, we have :

$$
\begin{aligned}
& p=(e+1)\left(k_{1}+k_{2}-1\right)+1+(e+1) x_{2}=(e+1)\left(k_{1}^{\prime}+k_{2}^{\prime}\right)+1, \\
& q=e\left(k_{1}+k_{2}-1\right)+1+e x_{2}=e\left(k_{1}^{\prime}+k_{2}^{\prime}\right)+1, \\
& r=(e+1)\left(k_{1}+k_{2}-1\right)-k_{1}+2+e x_{2}=p_{0}-k_{1}^{\prime} \text { and } \\
& s=-1 .
\end{aligned}
$$

Then by Theorem 1 of [6], we see that $T(p, q ; r, s)$ is a composite twisted torus knot if $x_{1}=1$. See Theorem 3.1 in the present paper. 
In [4], S. Y. Lee showed the following (c.f. [8]) :

Theorem 1.4 (Theorem 1 of [4]). Suppose $r=q k$ for some integer $k$. Then $T(p, q ; r, s)$ is the $\left(q, p+k^{2} q s\right)$-cable knot along the torus knot $T(k, k s+1)$.

Thus, by noting (3) of Theorem 1.1 and Remark 1.3, we conjecture the following :

Conjecture. A twisted torus knot $T(p, q ; r, s)$ has an essential torus in the exterior, if and olny if it is a knot in Theorem 1.1 or $r=q k$ for some integer $k$. In particular, $T(p, q ; r, s)$ is a composite knot if and only if it is a knot in Theorem 1.1 with $x_{1}=1$.

Throughout the present paper, we will work in the piecewise linear category. For a manifold $X$ and a subcomplex $Y$ in $X$, we denote a regular neighborhood of $Y$ in $X$ by $N(Y, X)$ or $N(Y)$ simply.

\section{Parallelized torus knots and parallelized twisted torus knots}

Let $T\left(p_{0}, q_{0}\right)$ be the torus knot of type $\left(p_{0}, q_{0}\right)$, where $p_{0}$ and $q_{0}$ are positive coprime integers with $p_{0}>1$, and let $x_{1}$ and $x_{2}$ be positive integers. Take four points $\mathrm{P}_{1}$, $\mathrm{P}_{2}, \mathrm{P}_{3}$ and $\mathrm{P}_{4}$ on the adjacent two strands in $T\left(p_{0}, q_{0}\right)$ as in Figure 3. Then replace the arc $\mathrm{P}_{1}$ through $\mathrm{P}_{3}$ with $x_{1}$ parallel strings and the $\operatorname{arc} \mathrm{P}_{2}$ through $\mathrm{P}_{4}$ with $x_{2}$ parallel strings. In addition, replace the rectangle $\mathrm{P}_{1} \mathrm{P}_{2} \mathrm{P}_{3} \mathrm{P}_{4}$ with $x_{1}+x_{2}$ strands as in Figure 4. Then we get a torus knot or a torus link $T(p, q)$ for some $p, q$.

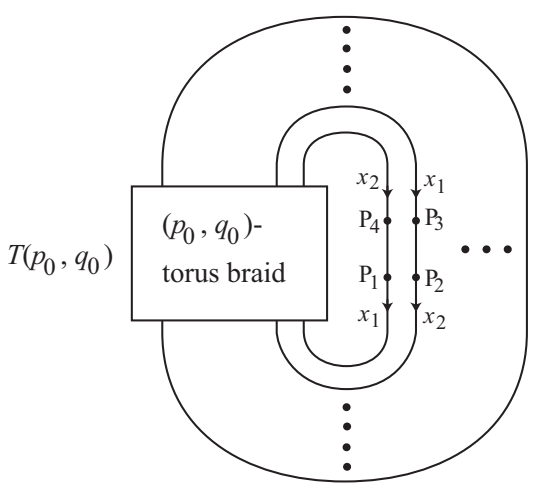

Figure 3

Let's detect $p$ and $q$. First, number the $p_{0}$ strings below the $\left(p_{0}, q_{0}\right)$ torus braid $0,1,2, \cdots, p_{0}-2, p_{0}-1$ as in Figure 5 . The arc starting at $\mathrm{P}_{1}$ goes into the braid at $p_{0}-1$ and goes out at $q_{0}-1$. After coming back round once it goes into the braid again and goes out at $2 q_{0}-1$. Next it goes out the braid at $3 q_{0}-1$. By continuing these procedures, it finally goes out at $a q_{0}-1 \equiv p_{0}-2\left(\bmod p_{0}\right)$. Then it meets the point $\mathrm{P}_{3}$. Hence we have $a q_{0} \equiv-1\left(\bmod p_{0}\right)$. Similarly the arc starting at $\mathrm{P}_{2}$ goes into the braid at $p_{0}-2$ and goes out at $q_{0}-2$. Then it goes out the braid at 


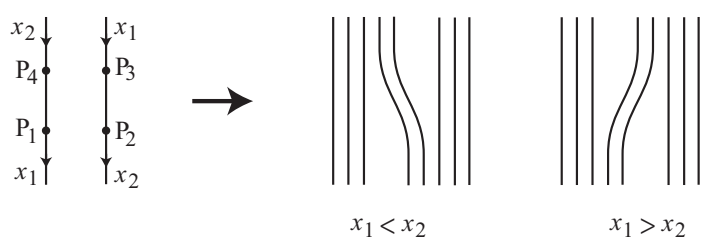

Figure 4

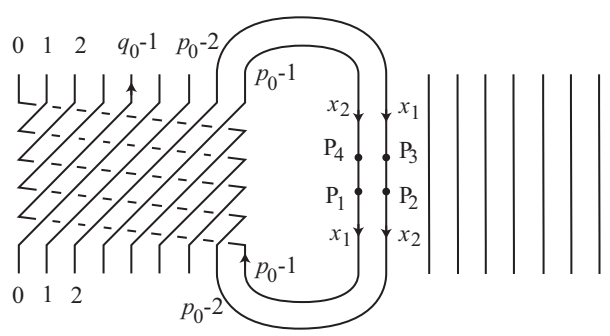

Figure 5

$2 q_{0}-2,3 q_{0}-2, \cdots$, and finally goes out at $b q_{0}-2 \equiv p_{0}-1\left(\bmod p_{0}\right)$. Then it meets the point $\mathrm{P}_{4}$. Hence we have $b q_{0} \equiv 1\left(\bmod p_{0}\right)$.

Thus we have $p=a x_{1}+b x_{2}$, where $a$ and $b$ are the least positive integers such that $a q_{0} \equiv-1\left(\bmod p_{0}\right), b q_{0} \equiv 1\left(\bmod p_{0}\right)$ and $a+b=p_{0}$.

By the similar arguments, we have $q=c x_{1}+d x_{2}$, where $c$ and $d$ are the least positive integers such that $c p_{0} \equiv 1\left(\bmod q_{0}\right), d p_{0} \equiv-1\left(\bmod q_{0}\right)$ and $c+d=q_{0}$.

In general, we have :

Proposition 2.1. For coprime positive integers $p_{0}$ and $q_{0}$, there uniquely exist positive integers $a, b, c, d$ which satisfy the following conditions :

$$
\text { (1) }\left\{\begin{array} { l } 
{ a + b = p _ { 0 } } \\
{ a q _ { 0 } \equiv - 1 ( \operatorname { m o d } p _ { 0 } ) } \\
{ b q _ { 0 } \equiv 1 ( \operatorname { m o d } p _ { 0 } ) }
\end{array} \quad \left\{\begin{array}{l}
c+d=q \\
c p_{0} \equiv 1\left(\bmod q_{0}\right) \\
d p_{0} \equiv-1\left(\bmod q_{0}\right)
\end{array}\right.\right.
$$

Proof. Consider the set $\left\{0, q_{0}, 2 q_{0}, \cdots,\left(p_{0}-1\right) q_{0}\right\}$. Then, by $\operatorname{gcd}\left(p_{0}, q_{0}\right)=1$, these $p_{0}$ integers are different to each other $\left(\bmod p_{0}\right)$. Then this set coincides with the set $\left\{0,1,2, \cdots, p_{0}-1\right\}\left(\bmod p_{0}\right)$, and hence there is only one integer $a$ with $a q_{0} \equiv p_{0}-1 \equiv-1\left(\bmod p_{0}\right)$. Then put $b=p_{0}-a$, and we have $b q_{0}=\left(p_{0}-a\right) q_{0}=$ $p_{0} q_{0}-a q_{0} \equiv 0-(-1)=1\left(\bmod p_{0}\right)$. This completes the proof of $(1)$, and $(2)$ is proved similarly.

Under the above situations, we have :

Proposition 2.2. Let $x_{1}$ and $x_{2}$ be positive integers, and put $p=a x_{1}+b x_{2}$ and 
$q=c x_{1}+d x_{2}$. Then $\operatorname{gcd}(p, q)=\operatorname{gcd}\left(x_{1}, x_{2}\right)$. In particular, $T(p, q)$ is a torus knot if and only if $\operatorname{gcd}\left(x_{1}, x_{2}\right)=1$.

Proof. Put $\operatorname{gcd}\left(x_{1}, x_{2}\right)=k$. Then we can put $x_{1}=k y_{1}, x_{2}=k y_{2}$, and $p=$ $k\left(a y_{1}+b y_{2}\right), q=k\left(c y_{1}+d y_{2}\right)$. Hence $\operatorname{gcd}(p, q) \geq k=\operatorname{gcd}\left(x_{1}, x_{2}\right)$.

Conversely, put $\operatorname{gcd}(p, q)=k$. Then we can put $p=k p_{1}, q=k q_{1}$.

Since $\left[\begin{array}{l}p \\ q\end{array}\right]=\left[\begin{array}{ll}a & b \\ c & d\end{array}\right]\left[\begin{array}{l}x_{1} \\ x_{2}\end{array}\right]$, we have :

$$
\left[\begin{array}{l}
x_{1} \\
x_{2}
\end{array}\right]=\frac{1}{a d-b c}\left[\begin{array}{rr}
d & -b \\
-c & a
\end{array}\right]\left[\begin{array}{l}
k p_{1} \\
k q_{1}
\end{array}\right] \cdots \text { (1) }
$$

Then $|a d-b c|<p_{0} q_{0}-1$ because $0<a, b<p_{0}$ and $0<c, d<q_{0}$. Moreover $a d-b c=a\left(q_{0}-c\right)-\left(p_{0}-a\right) c=a q_{0}-c p_{0} \equiv-1\left(\bmod p_{0}\right),\left(\bmod q_{0}\right)$. This implies that $a d-b c=-1$, and by (1) we have $\operatorname{gcd}\left(x_{1}, x_{2}\right) \geq k=\operatorname{gcd}(p, q)$. This completes the proof.

Let $T\left(p_{0}, q_{0} ; r_{0}, s_{0}\right)$ be a given twisted torus knot. Then by the same way as the case of torus knots, we can construct a parallelized twisted torus knot or a parallelized twisted torus link $T(p, q ; r, s)$. Then $p=a x_{1}+b x_{2}, q=c x_{1}+d x_{2}, r=r_{1} x_{1}+r_{2} x_{2}$ and $s=s_{0}$, where $a, b, c, d$ are those integers in Proposition 2.1 and $r_{1}, r_{2}$ are some positive integers with $r_{1}+r_{2}=r_{0}$. Then by Proposition 2.2, we see that $T(p, q ; r, s)$ is a knot if and only if $\operatorname{gcd}\left(x_{1}, x_{2}\right)=1$.

\section{Proof of Theorem 1.1}

Let $B$ be a 3-ball and $t^{1} \cup t^{2} \cup \cdots \cup t^{n} n$ arcs properly embedded in $B$, then we call the pair $\left(B, t^{1} \cup t^{2} \cup \cdots \cup t^{n}\right)$ an $n$-string tangle. We say that $\left(B, t^{1} \cup t^{2} \cup \cdots \cup t^{n}\right)$ is essential if $c l\left(\partial B-N\left(t^{1} \cup t^{2} \cup \cdots \cup t^{n}\right)\right)$ is incompressible in $\operatorname{cl}\left(B-N\left(t^{1} \cup t^{2} \cup \cdots \cup t^{n}\right)\right)$ if $n>1$, and $t^{1}$ is a knotted arc in $B$ if $n=1$, where $N\left(t^{1} \cup t^{2} \cup \cdots \cup t^{n}\right)$ is a regular neighborhood of $t^{1} \cup t^{2} \cup \cdots \cup t^{n}$ in $B$, and that the tangle is inessential if it is not essential. We say that a knot $K$ in the 3 -sphere $S^{3}$ has an $n$-string essential tangle decomposition if $\left(S^{3}, K\right)$ is decomposed into two $n$-string essential tangles $\left(B_{1}, t_{1}^{1} \cup t_{1}^{2} \cup \cdots \cup t_{1}^{n}\right) \cup\left(B_{2}, t_{2}^{1} \cup t_{2}^{2} \cup \cdots \cup t_{2}^{n}\right)$, and that the decomposition is inessential if it is not essential.

To prove Theorem 1.1, we construct parallelized twisted torus knots from composite twisted torus knots, and we will show that the decomposing 2-sphere of the connected sum becomes the decomposing 2-sphere of the tangle decomposition. In [6], we have shown the following:

Theorem 3.1 (Theorem 1 of [6]). Let $e>0, k_{1}>1, k_{2}>1$ be integers, and put $p_{0}=(e+1)\left(k_{1}+k_{2}\right)+1, q_{0}=e\left(k_{1}+k_{2}\right)+1, r_{0}=p_{0}-k_{1}$ and $s_{0}=-1$. Then 
$T\left(p_{0}, q_{0} ; r_{0}, s_{0}\right)$ is the connected sum of $T\left(k_{1}, e k_{1}+1\right)$ and $T\left(k_{2},-(e+1) k_{2}-1\right)$.

To get parallelized twisted torus knots from the above composite knots, first we calculate the integers $a, b, c, d$ in Proposition 2.1 to get $p$ and $q$.

Proposition 3.2. For $p_{0}=(e+1)\left(k_{1}+k_{2}\right)+1$ and $q_{0}=e\left(k_{1}+k_{2}\right)+1$, those integers $a, b, c, d$ in Proposition 2.1 are as follows :

$a=(e+1)\left(k_{1}+k_{2}-1\right)+1, b=e+1, c=e\left(k_{1}+k_{2}-1\right)+1$ and $d=e$.

Proof. First we have $b=e+1$, because $(e+1) q_{0}=(e+1)\left(e\left(k_{1}+k_{2}\right)+1\right)=$ $(e+1) e\left(k_{1}+k_{2}\right)+e+1=e\left((e+1)\left(k_{1}+k_{2}\right)+1\right)+1=e p_{0}+1 \equiv 1\left(\bmod p_{0}\right)$. Then $a=p_{0}-b=(e+1)\left(k_{1}+k_{2}\right)+1-(e+1)=(e+1)\left(k_{1}+k_{2}-1\right)+1$ and $a q_{0}=\left(p_{0}-b\right) q_{0}=p_{0} q_{0}-b q_{0} \equiv-1\left(\bmod p_{0}\right)$.

Next we have $d=e$ because $e p_{0}=e\left((e+1)\left(k_{1}+k_{2}\right)+1\right)=e(e+1)\left(k_{1}+k_{2}\right)+e=$ $(e+1) e\left(k_{1}+k_{2}\right)+e+1-1=(e+1)\left(e\left(k_{1}+k_{2}\right)+1\right)-1=(e+1) q-1 \equiv-1\left(\bmod q_{0}\right)$. Then $c=q_{0}-d=e\left(k_{1}+k_{2}\right)+1-e=e\left(k_{1}+k_{2}-1\right)+1$ and $c p_{0}=\left(q_{0}-d\right) p_{0}=q_{0} p_{0}-d p_{0} \equiv 1$ $\left(\bmod q_{0}\right)$. This completes the proof.

To calculate $r$ and to get concrete expression of the tangle decompositions, consider the twisted torus knots in Theorem 3.1. Put $K_{0}=T\left(p_{0}, q_{0} ; r_{0}, s_{0}\right), K_{1}=T\left(k_{1}, e k_{1}+\right.$ 1) and $K_{2}=T\left(k_{2},-(e+1) k_{2}-1\right)$, then $K_{0}=K_{1} \# K_{2}$. Let $V$ be a standard genus two handlebody in $S^{3}$, and put $\partial V=F$. Then, since any twisted torus knot can be embedded in $F$ in a standard way, we may assume that $K_{0}$ is in $F$. Let $S$ be the decomposing 2-sphere of the connected sum $K_{0}=K_{1} \# K_{2}$, then, by the proof in [6] of Theorem 3.1, we may assume that $S$ intersects $V$ in a separating disk and that $S \cap F$ is a single loop. Then, by noting that $p_{0}-r_{0}=k_{1}, S \cap F$ runs along the both sides of $k_{1}$ strings and $(S \cap F) \cap K_{0}$ consists of the two points $\mathrm{Q}_{1}$ and $\mathrm{Q}_{2}$ as in Figure 6 , where Figure 6 is the case of $e=1, k_{1}=3, k_{2}=2$ and the connected sum is $T(11,6 ; 8,-1)=T(3,4) \# T(2,-5)$.

We split $K_{0}$ at $\mathrm{Q}_{1}, \mathrm{Q}_{2}$ into two arcs, and to get $K_{1}$ and $K_{2}$, connect the two points with the arc in the disk $S \cap V$. First we consider $K_{1}$ as in Figure $7\left(k_{1}=5\right)$. Then, by noting that $p_{0}-r_{0}=k_{1}$ and $K_{1}=T\left(k_{1}, e k_{1}+1\right)$, we see that the arc $\mathrm{P}_{2} \mathrm{P}_{4}$ is contained in $K_{1}$, and hence exactly one string of $k_{1}$ strings is replaced with $x_{2}$ parallel strings. Then the other $\left(k_{1}-1\right)$ strings are contained in the $\operatorname{arc} \mathrm{P}_{1} \mathrm{P}_{3}$ and are replaced with $x_{1}$ parallel strings. Thus we get the torus knot $T\left(\left(k_{1}-1\right) x_{1}+x_{2}, e\left(\left(k_{1}-1\right) x_{1}+x_{2}\right)+x_{1}\right)$, and this torus knot intersects the original decomposing 2-sphere in $x_{1}$ points at each $\mathrm{Q}_{i}(i=1,2)$. This implies that $r=p-\left(\left(k_{1}-1\right) x_{1}+x_{2}\right)$.

For the knot $K_{2}$, by the above arguments, we see that the whole string of $K_{2}$ is contained in the arc $\mathrm{P}_{1} \mathrm{P}_{3}$. Hence by replacing the whole string with $x_{1}$ parallel strings, we get the torus link $T\left(k_{2} x_{1},-\left((e+1) k_{2}+1\right) x_{1}\right)$. This torus link intersects the original decomposing 2 -sphere in $x_{1}$ points at each $\mathrm{Q}_{i}(i=1,2)$ similarly to the 


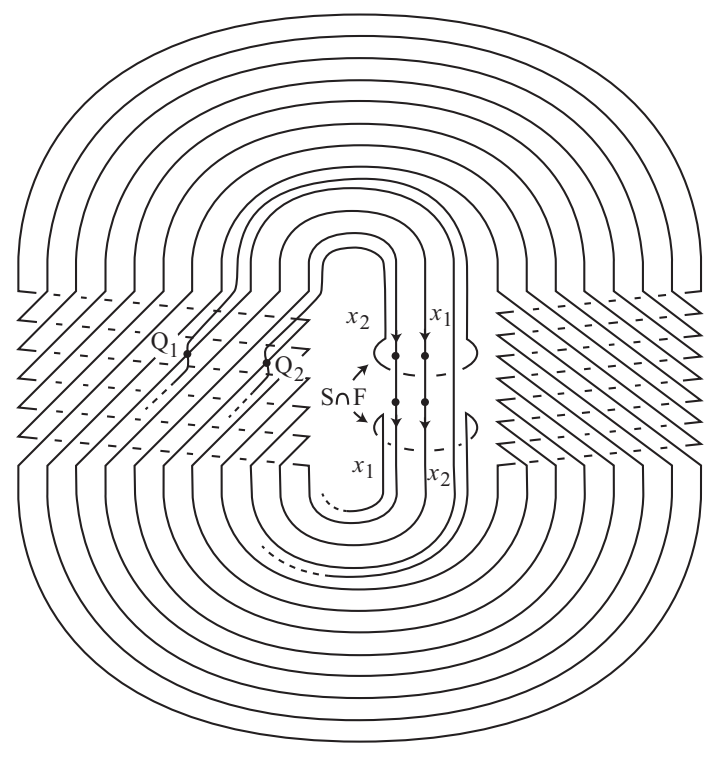

Figure 6

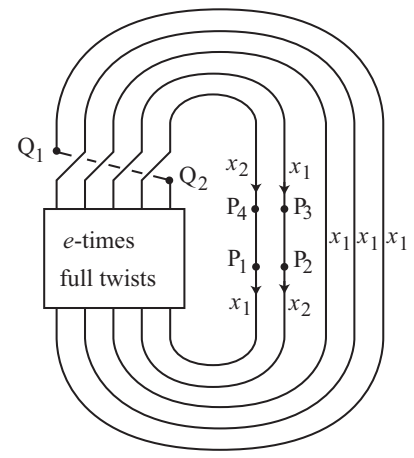

Figure 7 
case of $K_{1}$.

By summarizing the above calculations, we have the following, and get the knots in Theorem 1.1.

$$
\begin{aligned}
& p=a x_{1}+b x_{2}=\left((e+1)\left(k_{1}+k_{2}-1\right)+1\right) x_{1}+(e+1) x_{2} \\
& q=c x_{1}+d x_{2}=\left(e\left(k_{1}+k_{2}-1\right)+1\right) x_{1}+e x_{2} \\
& r=p-\left(\left(k_{1}-1\right) x_{1}+x_{2}\right)=\left((e+1)\left(k_{1}+k_{2}-1\right)+1\right) x_{1}+(e+1) x_{2}-\left(\left(k_{1}-1\right) x_{1}+x_{2}\right) \\
& \quad=\left((e+1)\left(k_{1}+k_{2}-1\right)-k_{1}+2\right) x_{1}+e x_{2} \\
& s=s_{0}=-1
\end{aligned}
$$

Finally, we need to show that the above tangle decompositions are all essential. If $x_{1}=1$, then the decompositions are the connected sums and are all essential because both $k_{1}$ and $k_{2}$ are greater than one and factor knots are non-trivial knots.

Suppose $x_{1}>1$. By the definition of tangles, we see that an $n$-string tangle $\left(B, t^{1} \cup\right.$ $t^{2} \cup \cdots \cup t^{n}$ ) with $n>1$ is essential if and only if there is no disk properly embedded in $B$ which separates the $\operatorname{arcs} t^{1} \cup t^{2} \cup \cdots \cup t^{n}$. From this view point, in the next section, we will show that both of $x_{1}$-string tangles constructed from the torus knot $T\left(\left(k_{1}-1\right) x_{1}+x_{2}, e\left(\left(k_{1}-1\right) x_{1}+x_{2}\right)+x_{1}\right)$ and the torus link $T\left(k_{2} x_{1},-\left((e+1) k_{2}+1\right) x_{1}\right)$ are essential (Proposition 4.3).

In addition, by tubing the decomposing 2 -sphere along the torus link $T\left(k_{2} x_{1},-((e+\right.$ 1) $\left.\left.k_{2}+1\right) x_{1}\right)$, we have an essential torus whose companion is the torus $\operatorname{knot} T\left(k_{2},-(e+\right.$ 1) $\left.k_{2}-1\right)$. This completes the proof of Theorem 1.1.

Example 3.3. Put $e=1, k_{1}=k_{2}=2$, and let $x_{1}, x_{2}$ be positive integers. Then by the above arguments, $p=7 x_{1}+2 x_{2}, q=4 x_{1}+x_{2}, r=6 x_{1}+x_{2}$ and $T(p, q ; r,-1)$ is the $x_{1}$-string fusion of $T\left(x_{1}+x_{2}, 2 x_{1}+x_{2}\right)$ and $T\left(2 x_{1},-5 x_{1}\right)$. Hence by putting $x_{1}=2, x_{2}=3$, we see that $T(20,11 ; 15,-1)$ is the 2 -string fusion of $T(5,7)$ and $T(4,-10)$, and this is the example in Introduction. We note that there may be ambiguity on twists in the fusion. But by more detailed arguments we get Figure 2 . If we put $x_{1}=2$ and $x_{2}=1$, then we see that $T(16,9 ; 13,-1)$ is the 2 -string fusion of $T(3,5)$ and $T(4,-10)$. This is the smallest example of our knots.

\section{Essential tangles}

Let $p, q$ be coprime integers with $2<p<q$, and $k$ an integer with $1<k<p$. Consider the torus knot $T(p, q)$ and take an $\operatorname{arc} \alpha$ which intersects $k$ strings in the parallel $p$ strings of $T(p, q)$ as in Figure 8(1). Let $N(\alpha)$ be a regular neighborhood of $\alpha$ in $S^{3}$. Put $B=\operatorname{cl}\left(S^{3}-N(\alpha)\right)$ and $t(p, q ; k)=c l(T(p, q)-N(\alpha))$. Then the pair $(B, t(p, q ; k))$ is a $k$-string tangle as in Figure $8(2)$.

Lemma 4.1. If $k=2$, then the tangle $(B, t(p, q ; 2))$ is an essential tangle.

Proof. Put $t(p, q ; 2)=t_{1} \cup t_{2}$. We first show that at least one of $t_{1}$ and $t_{2}$ is a knotted arc in $B$. By $p<q$, we can put $q=n p+m(0<m<p)$. Then, since 


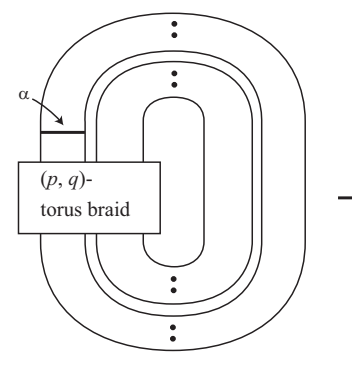

(1)

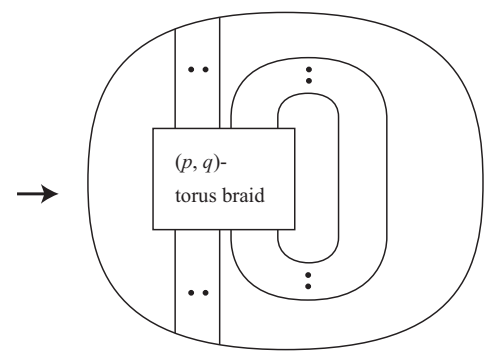

(2)

Figure 8

$t_{1}$ and $t_{2}$ are arcs properly embedded in $B$ each of which is a local torus knot, we can put $t_{1}=T(a, n a+c)$ and $t_{2}=T(b, n b+d)$, where $a+b=p$ and $c+d=m$ by the arguments similar to those in Section 2. We note that $(n a+c)+(n b+d)=$ $n(a+b)+(c+d)=n p+m=q$. Then, by $a \leq n a+c, b \leq n b+d$ and $a+b=p>2$, we see that at least one of $t_{1}$ and $t_{2}$ is a knotted arc in $B$.

Suppose the tangle $(B, t(p, q ; 2))$ is inessential. Then, by the definition of essential tangles, there is a disk properly embedded in $B$ which separates $t_{1}$ and $t_{2}$. Hence $\operatorname{cl}\left(B-N\left(t_{1} \cup t_{2}\right)\right)$ is not a handlebody. However, by [1], the arc $\alpha$ connecting the adjacent strings is an unknotting tunnel of $T(p, q)$, and hence $\operatorname{cl}\left(B-N\left(t_{1} \cup t_{2}\right)\right)$ is a handlebody. This is a contradiction, and completes the proof.

Lemma 4.2. $(B, t(p, q ; k))$ has exactly two parallel classes of strings.

Proof. First suppose $k=3$, and number the 3 strings under the torus braid 0,1 and 2 . Then the 3 strings above the torus braid has the three cases, $(0,1,2),(2,0$, $1)$ and $(1,2,0)$. However if it is $(0,1,2)$, then $T(p, q)$ is a 3 -component link. Hence we have the two cases $(2,0,1)$ and $(1,2,0)$ as in Figure 9 . Then we can see that the adjacent two strings $(0,1)$ or $(1,2)$ are mutually parallel strings.
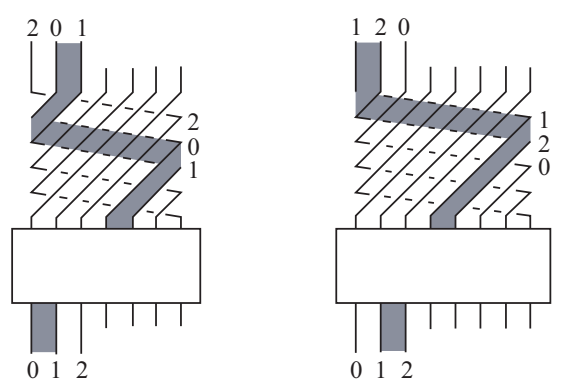

Figure 9

Next suppose $k>3$. Then by the arguments similar to the case of $k=3$, we 
see that there are adjacent strings which are mutually parallel. Then by inductive arguments, we see that the parallel classes of the strings consists of two classes.

Proposition 4.3. The tangle $(B, t(p, q ; k))$ is an essential tangle.

Proof. By Lemma 4.2, $t(p, q ; k)=t_{1} \cup t_{2} \cup \cdots \cup t_{k}$ has two parallel classes. Hence we may assume that there are two rectangles $R_{1}, R_{2}$ in $B$ such that $t_{1} \cup \cdots \cup t_{i} \subset R_{1}$ and $t_{i+1} \cup \cdots \cup t_{k} \subset R_{2}$, where $\partial R_{1}$ consists of $t_{1} \cup t_{i} \cup\left(\right.$ two $\operatorname{arcs}$ in $\partial B$ ) and $\partial R_{2}$ consists of $t_{i+1} \cup t_{k} \cup($ two $\operatorname{arcs}$ in $\partial B)$.

Suppose the tangle $(B, t(p, q ; k))$ is inessential. Then, by the definition of essential tangles, there is a disk, say $D$, properly embedded in $B$ which separates $t_{1} \cup \cdots \cup t_{k}$. Suppose $R_{1} \cap D \neq \emptyset$. Then by standard cut and paste operations and by replacing disks if necessary, we may assume that each component of $R_{1} \cap D$ is an arc parallel to the strings $t_{1} \cup t_{i}$. This means that those strings are all unknotted. Then by some cut and paste operations, we can get another separating disk, say $D^{\prime}$, with $D^{\prime} \cap R_{1}=\emptyset$. If $R_{2} \cap D \neq \emptyset$, we can remove the intersections similarly.

Hence we may assume that $\left(R_{1} \cup R_{2}\right) \cap D=\emptyset$ and that $D$ separates the two rectangles $R_{1}$ and $R_{2}$. Then by the arguments similar to those in the proof of Lemma 4.1, we have a contradiction. This completes the proof.

\section{References}

[1] M. Boileau, M. Rost and H. Zieschang, On Heegaard decompositions of torus knot exteriors and related Seifert fibre spaces, Math. Ann. 279 (1988) 553-581.

[2] P. J. Callahan, J. C. Dean and J. R.Weeks, The simplest hyperbolic knots, J. Knot Theory Ramifications, 8 (1999) 279-297.

[3] J. H. Lee, Twisted torus knots $T(p, q ; 3, s)$ are tunnel number one, J. Knot Theory Ramifications, 20 (2011) 807-811.

[4] S. Y. Lee, Twisted torus knots $T(p, q ; k q, s)$ are cable knots, J. Knot Theory Ramifications, 21 (2012) 1250005(1-4).

[5] K. Morimoto, Essential surfaces in the exteriors of torus knots with twists on 2-strands, preprint.

[6] K. Morimoto, On composite twisted torus knots, to appear in Tokyo Journal of Mathematics.

[7] K. Morimoto, M. Sakuma and Y. Yokota, Examples of tunnel number one knots which have the property " $1+1=3$ ", Math. Proc. Camb. Phil. Soc. 119 (1996) 113-118.

[8] K. Morimoto and Y. Yamada, A note on essential tori in the exteriors of torus knots with twists, Kobe J. Math., 26 (2009) 29-34.

[9] D. Rolfsen, Knots and Links, AMS Chelsea Publishing (2003). 